\title{
Some Reliability Measures of a Deteriorating System
}

\author{
Ibrahim Yusuf*, Nafiu Hussaini, Bashir M. Yakasai \\ Department of Mathematical Sciences, Bayero University, Kano, Nigeria \\ *Corresponding author E-mail: ibrahimyusif@yahoo.com
}

Copyright $\odot 2014$ Ibrahim Yusuf et al. This is an open access article distributed under the Creative Commons Attribution License, which permits unrestricted use, distribution, and reproduction in any medium, provided the original work is properly cited.

\begin{abstract}
In this paper, we studied some reliability characteristics of a repairable 3-out-of-4 system with three modes: normal, deterioration and failure modes. The deterioration mode could be slow or fast. Failure and repair time are assumed to be exponential. We develop the explicit expressions for steady-state availability, busy period of repair man, profit function and mean time to system failure for the system using linear first order differential equations. Various cases are analyzed graphically to investigate the impact of system parameters on MTSF and profit function.
\end{abstract}

Keywords: Reliability, availability, profit, deterioration.

\section{Introduction}

During operation the strengths of systems are gradually deteriorated, until some point of deterioration failure, or other types of failures. Minor and major minimal maintenance are vital in the analysis of deteriorating systems as they help in improving reliability and availability of the systems. The system in this study consists of four units in which three units are sufficient to perform the entire function of the system. This system has wide application in the real world especially in industries. For example, [1], analyzed reliability models for 2-out-of-3 redundant system subject to conditional arrival time of the server and [2] present reliability and economic analysis of 2-out-of-3 redundant system with priority to repair, while [3] studied MTSF and cost effectiveness of 2-out-of-3 cold standby system with probability of repair and inspection. Reference [4] examined the cost benefit analysis of series systems with cold standby components and repairable service station. Reference [5], [6] examined the cost analysis of two unit cold standby system involving preventive maintenance respectively. Reference [7] studied the cost and probabilistic analysis of series system with mixed standby components, and [8] studied cost benefit analysis of series systems with warm standby components involving general repair time where the server is not subject to breakdowns. The failure time and repair time are assumed to have exponential distribution. Measures of system effectiveness such MTSF, steady-state availability, busy period and profit function are obtained. Reference [9] studied availability and profit of a repairable 3-out-of-4 system with preventive maintenance, while [10] evaluate the reliability of network flows with stochastic capacity and cost constraint.

In this paper, a two-stage deteriorating linear 3-out-of-4 system is constructed and derived its corresponding mathematical models. The main contribution of this paper is twofold. First, is to develop the explicit expressions for system availability, busy period, profit function and mean time to system failure. The second is to perform a parametric investigation of various system parameters on mean time to system failure and profit function and capture their effect. The rest of the paper is organized as follows. Section 2 is the description and states of the system. Section 3 deals with models formulation. The results of our numerical simulations are presented and discussed in Section 4. The paper is concluded in Section 5.

\section{Description of the system}

We consider a linear consecutive 3-out-of-4 system with three modes: operation, deterioration and failure modes. The deterioration mode could be slow or fast. It is assumed that the system transits from operation to slow or fast deterioration with rate $\delta_{1}$ and $\delta_{2}$ respectively. It is also assumed that the two consecutive units never fail 
simultaneously. Minor minimal maintenance is invoke with rate $\mu_{1}$ to regain the system to its early stage prior to slow deterioration or major minimal maintenance will be done with rate $\mu_{2}$ to regain the system to its early stage prior to fast deterioration. Unit I fail with rate $\beta_{1}$ and am under minimal repair with rate $\alpha_{1}$ and unit III is switch on. It is assumed that the switch from standby to operation is perfect. Similarly, unit II fails with rate $\beta_{2}$ and is minimally repaired with rate $\alpha_{2}$. The system failed when unit I and II have failed. The system is attended by two repair men.

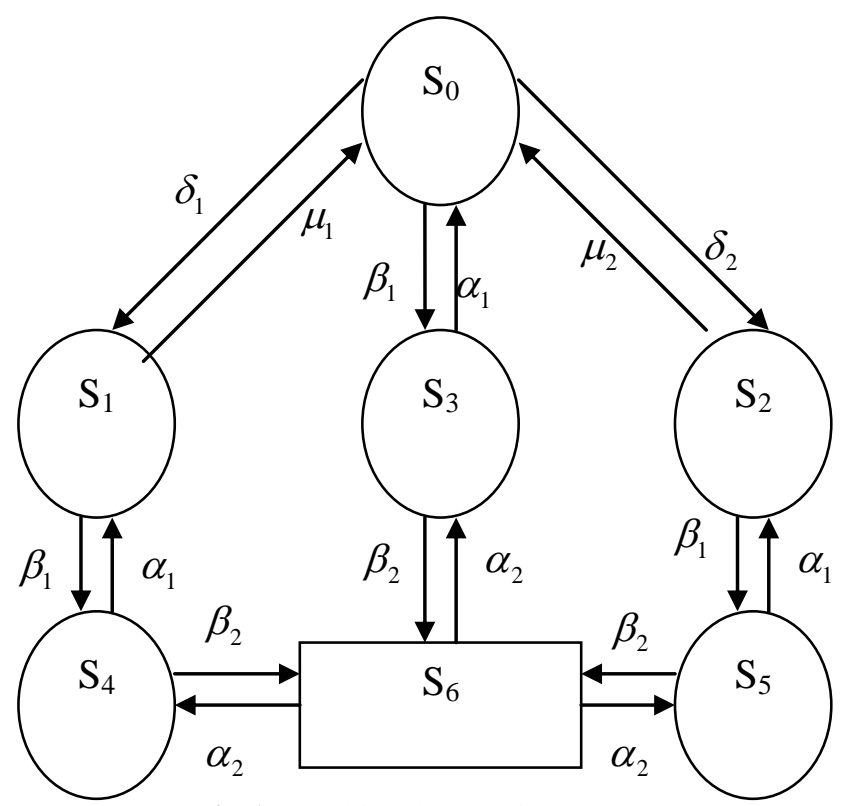

Fig. 1: Transition diagram of the system

\section{States of the System}

State $S_{0}$ : Units I, II and III are in operation, unit IV is in standby, the system is operational

State $S_{1}$ : The system is under slow deterioration and is receiving minor minimal maintenance and is operational

State $S_{2}$ : The system is under fast deterioration and is receiving major minimal maintenance and is operational

State $S_{3}$ : Unit I failed and am under repair, units II, III and IV are in operation, the system is Operational

State $S_{4}$ : Unit I failed and is under repair, units II, III and IV are in operation, the system is in slow deterioration stage and Operational

State $S_{5}$ : Unit I failed and is under repair, units II, III and IV are in operation, the system is in fast deterioration stage and Operational

State $S_{6}$ : Units I and II failed and are under repair, III and IV are idle, the system failed

\section{Models formulation}

Let $P(t)$ be the probability row vector at time $t$, then the initial conditions for this problem are as follows:

$P(0)=\left[P_{0}(0), P_{1}(0), P_{2}(0), P_{3}(0), P_{4}(0), P_{5}(0), P_{6}(0)\right]=[1,0,0,0,0,0,0]$

We obtain the following system of differential equations:

$P_{0}^{\prime}(t)=-\left(\delta_{1}+\delta_{2}+\beta_{1}\right) P_{0}(t)+\mu_{1} P_{1}(t)+\mu_{2} P_{2}(t)+\alpha_{1} P_{3}(t)$

$P_{1}^{\prime}(t)=-\left(\mu_{1}+\beta_{1}\right) P_{1}(t)+\delta_{1} P_{0}(t)+\alpha_{1} P_{4}(t)$

$P_{2}^{\prime}(t)=-\left(\mu_{2}+\beta_{1}\right) P_{2}(t)+\delta_{2} P_{0}(t)+\alpha_{1} P_{5}(t)$

$P_{3}^{\prime}(t)=-\left(\alpha_{1}+\beta_{2}\right) P_{3}(t)+\beta_{1} P_{0}(t)+\alpha_{2} P_{6}(t)$

$P_{4}^{\prime}(t)=-\left(\alpha_{1}+\beta_{2}\right) P_{4}(t)+\beta_{1} P_{1}(t)+\alpha_{2} P_{6}(t)$

$P_{5}^{\prime}(t)=-\left(\alpha_{1}+\beta_{2}\right) P_{5}(t)+\beta_{1} P_{2}(t)+\alpha_{2} P_{6}(t)$

$P_{6}^{\prime}(t)=-3 \alpha_{2} P_{6}(t)+\beta_{2} P_{3}(t)+\beta_{2} P_{4}(t)+\beta_{2} P_{5}(t)$

The differential equations in (1) above is transformed into matrix as $P^{\prime}=T P$ 
Where

$T=\left[\begin{array}{ccccccc}-\left(\delta_{1}+\delta_{2}+\beta_{1}\right) & \mu_{1} & \mu_{2} & \alpha_{1} & 0 & 0 & 0 \\ \delta_{1} & -\left(\beta_{1}+\mu_{1}\right) & 0 & 0 & \alpha_{1} & 0 & 0 \\ \delta_{2} & 0 & -\left(\beta_{1}+\mu_{2}\right) & 0 & 0 & \alpha_{1} & 0 \\ \beta_{1} & 0 & 0 & -\left(\beta_{2}+\alpha_{1}\right) & 0 & 0 & \alpha_{2} \\ 0 & \beta_{1} & 0 & 0 & -\left(\beta_{2}+\alpha_{1}\right) & 0 & \alpha_{2} \\ 0 & 0 & \beta_{1} & 0 & 0 & -\left(\beta_{2}+\alpha_{1}\right) & \alpha_{2} \\ 0 & 0 & 0 & \beta_{2} & \beta_{2} & \beta_{2} & -3 \alpha_{2}\end{array}\right]$

\subsection{System availability and busy period analysis}

For the availability case of Fig. 1 using the initial condition in section 3 for this system, $P(0)=\left[P_{0}(0), P_{1}(0), P_{2}(0), P_{3}(0), P_{4}(0), P_{5}(0), P_{6}(0)\right]=[1,0,0,0,0,0,0]$

The system of differential equations in (1) for the system above can be expressed in matrix form as:

$\left[\begin{array}{c}P_{0}^{\prime}(t) \\ P_{1}^{\prime}(t) \\ P_{2}^{\prime}(t) \\ P_{3}^{\prime}(t) \\ P_{4}^{\prime}(t) \\ P_{5}^{\prime}(t) \\ P_{6}^{\prime}(t)\end{array}\right]=\left[\begin{array}{ccccccc}-\left(\delta_{1}+\delta_{2}+\beta_{1}\right) & \mu_{1} & \mu_{2} & \alpha_{1} & 0 & 0 & 0 \\ \delta_{1} & -\left(\beta_{1}+\mu_{1}\right) & 0 & 0 & \alpha_{1} & 0 & 0 \\ \delta_{2} & 0 & -\left(\beta_{1}+\mu_{2}\right) & 0 & 0 & \alpha_{1} & 0 \\ \beta_{1} & 0 & 0 & -\left(\beta_{2}+\alpha_{1}\right) & 0 & 0 & \alpha_{2} \\ 0 & \beta_{1} & 0 & 0 & -\left(\beta_{2}+\alpha_{1}\right) & 0 & \alpha_{2} \\ 0 & 0 & \beta_{1} & 0 & 0 & -\left(\beta_{2}+\alpha_{1}\right) & \alpha_{2} \\ 0 & 0 & 0 & \beta_{2} & \beta_{2} & \beta_{2} & -3 \alpha_{2}\end{array}\right]\left[\begin{array}{c}P_{9}(t) \\ P_{1}(t) \\ P_{2}(t) \\ P_{3}(t) \\ P_{4}(t) \\ P_{5}(t) \\ P_{6}(t)\end{array}\right]$

In steady state, the derivatives of state probabilities become zero, thus (2) becomes

$T P(\infty)=0$

Which in matrix form is?

$\left[\begin{array}{ccccccc}-\left(\delta_{1}+\delta_{2}+\beta_{1}\right) & \mu_{1} & \mu_{2} & \alpha_{1} & 0 & 0 & 0 \\ \delta_{1} & -\left(\beta_{1}+\mu_{1}\right) & 0 & 0 & \alpha_{1} & 0 & 0 \\ \delta_{2} & 0 & -\left(\beta_{1}+\mu_{2}\right) & 0 & 0 & \alpha_{1} & 0 \\ \beta_{1} & 0 & 0 & -\left(\beta_{2}+\alpha_{1}\right) & 0 & 0 & \alpha_{2} \\ 0 & \beta_{1} & 0 & 0 & -\left(\beta_{2}+\alpha_{1}\right) & 0 & \alpha_{2} \\ 0 & 0 & \beta_{1} & 0 & 0 & -\left(\beta_{2}+\alpha_{1}\right) & \alpha_{2} \\ 0 & 0 & 0 & \beta_{2} & \beta_{2} & \beta_{2} & -3 \alpha_{2}\end{array}\right]\left[\begin{array}{c}P_{9}(t) \\ P_{1}(t) \\ P_{2}(t) \\ P_{3}(t) \\ P_{4}(t) \\ P_{5}(t) \\ P_{6}(t)\end{array}\right]=\left[\begin{array}{c}0 \\ 0 \\ 0 \\ 0 \\ 0 \\ 0 \\ 0\end{array}\right]$

Using the normalizing condition

$P_{0}(\infty)+P_{1}(\infty)+P_{2}(\infty)+P_{3}(\infty)+P_{4}(\infty)+P_{5}(\infty)+P_{6}(\infty)=1$

We substitute (4) in the last row of (3). The resulting matrix is

$\left[\begin{array}{ccccccc}-\left(\delta_{1}+\delta_{2}+\beta_{1}\right) & \mu_{1} & \mu_{2} & \alpha_{1} & 0 & 0 & 0 \\ \delta_{1} & -\left(\beta_{1}+\mu_{1}\right) & 0 & 0 & \alpha_{1} & 0 & 0 \\ \delta_{2} & 0 & -\left(\beta_{1}+\mu_{2}\right) & 0 & 0 & \alpha_{1} & 0 \\ \beta_{1} & 0 & 0 & -\left(\beta_{2}+\alpha_{1}\right) & 0 & 0 & \alpha_{2} \\ 0 & \beta_{1} & 0 & 0 & -\left(\beta_{2}+\alpha_{1}\right) & 0 & \alpha_{2} \\ 0 & 0 & \beta_{1} & 0 & 0 & -\left(\beta_{2}+\alpha_{1}\right) & \alpha_{2} \\ 1 & 1 & 1 & 1 & 1 & 1 & 1\end{array}\right]\left[\begin{array}{c}P_{9}(t) \\ P_{1}(t) \\ P_{2}(t) \\ P_{3}(t) \\ P_{4}(t) \\ P_{5}(t) \\ P_{6}(t)\end{array}\right]=\left[\begin{array}{l}0 \\ 0 \\ 0 \\ 0 \\ 0 \\ 0 \\ 1\end{array}\right]$

Let $V$ be the time to failure of the system. The steady-state availability is given by:

$A_{v}(\infty)=P_{0}(\infty)+P_{1}(\infty)+P_{2}(\infty)+P_{3}(\infty)+P_{4}(\infty)+P_{5}(\infty)=\frac{N_{1}}{D_{1}}$

The steady-state busy period is given by:

$B_{V}(\infty)=P_{1}(\infty)+P_{2}(\infty)+P_{3}(\infty)+P_{4}(\infty)+P_{5}(\infty)+P_{6}(\infty)=\frac{N_{2}}{D_{1}}$ 


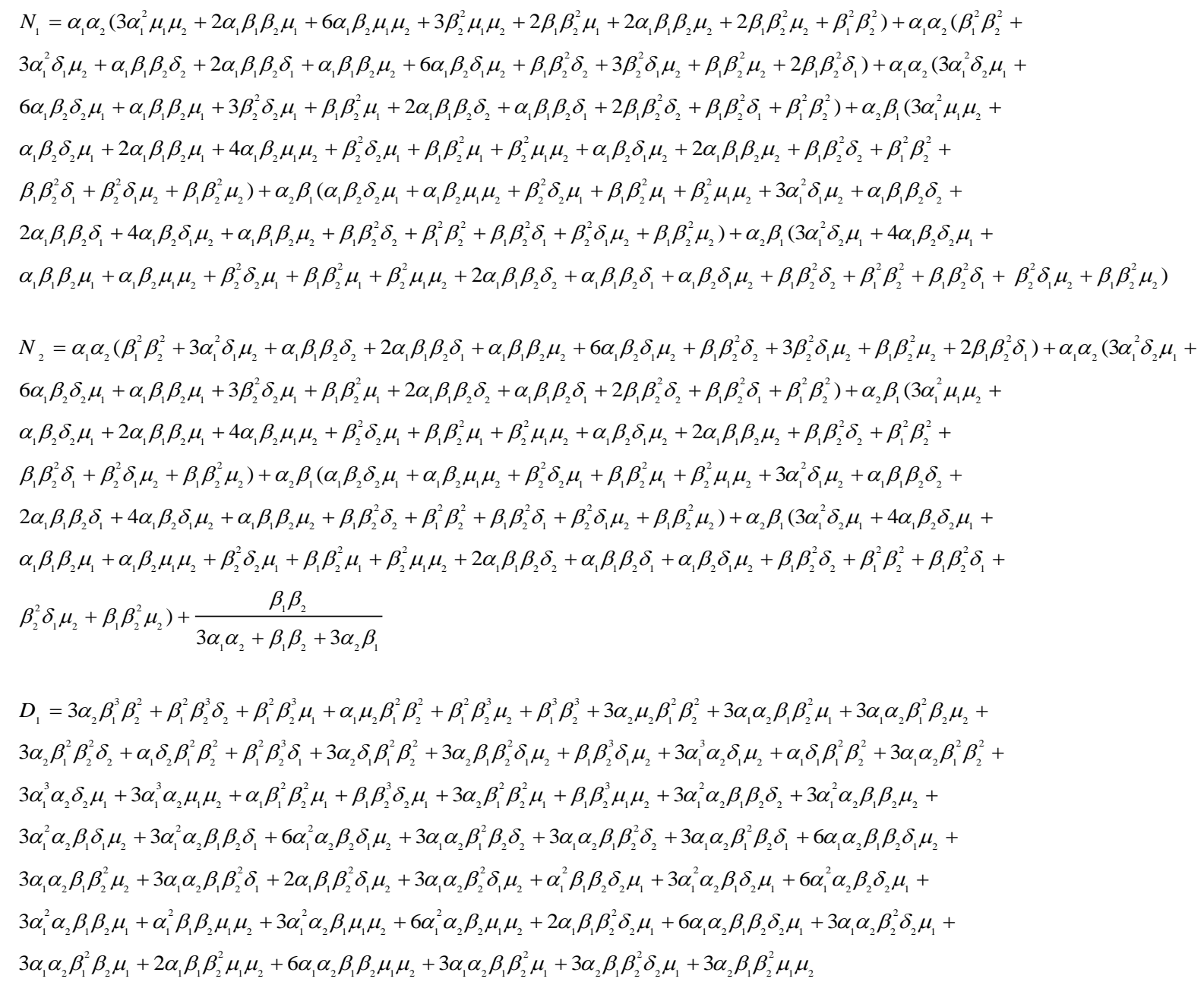

\subsection{Profit analysis}

The system is under minimal and major maintenance in states 1 and 2, while the units are under corrective maintenance at failure as can be observed in states 3, 4, 5 and 6. From Fig. 1 the repairman is busy performing minimal and major maintenance in states 1 and 2, and corrective maintenance in states 3, 4, 5 and 6. The expected profit per unit time incurred to the system in the steady-state is given by:

Profit $=$ total revenue generated - accumulated cost incurred due to maintenance at deterioration and repair due failure.

$P F=C_{0} A_{v}(\infty)-C_{1} B_{v}(\infty)$

Where $P F:$ is the profit incurred to the system

$C_{0}:$ is the revenue per unit up time of the system

$C_{1}$ : is the cost per unit time which the system is under maintenance and repair

\subsection{Mean time to system failure analysis}

It is difficult to evaluate the transient solutions, hence we follow [2], [3], [5], the procedure to develop the explicit expression for MTSF is to delete the seventh row and column of matrix $T$ and take the transpose to produce a new matrix, say $Q$. The expected time to reach an absorbing state is obtained from

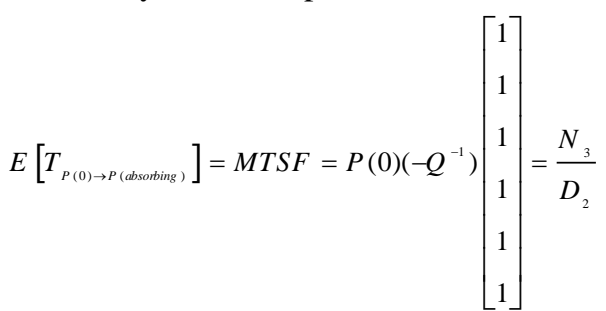

Where 
$Q=\left[\begin{array}{cccccc}-\left(\delta_{1}+\delta_{2}+\beta_{1}\right) & \delta_{1} & \delta_{2} & \beta_{1} & 0 & 0 \\ \mu_{1} & -\left(\beta_{1}+\mu_{1}\right) & 0 & 0 & \beta_{1} & 0 \\ \mu_{2} & 0 & -\left(\beta_{1}+\mu_{2}\right) & 0 & 0 & \beta_{1} \\ \alpha_{1} & 0 & 0 & -\left(\beta_{2}+\alpha_{1}\right) & 0 & 0 \\ 0 & \alpha_{1} & 0 & 0 & -\left(\beta_{2}+\alpha_{1}\right) & 0 \\ 0 & 0 & \alpha_{1} & 0 & 0 & -\left(\beta_{2}+\alpha_{1}\right)\end{array}\right]$

$N_{3}=\beta_{1} \beta_{2}\left(\alpha_{1}^{3} \mu_{1} \mu_{2}+\alpha_{1}^{2} \beta_{1} \beta_{2} \mu_{1}+3 \alpha_{1}^{2} \beta_{2} \mu_{1} \mu_{2}+2 \alpha_{1} \beta_{1} \beta_{2}^{2} \mu_{1}+3 \alpha_{1} \beta_{2}^{2} \mu_{1} \mu_{2}+\beta_{1} \beta_{2}^{3} \mu_{1}+\beta_{2}^{3} \mu_{1} \mu_{2}+\alpha_{1}^{2} \beta_{1} \beta_{2} \mu_{2}+\alpha_{1} \beta_{1}^{2} \beta_{2}^{2}+\right.$

$\left.2 \alpha_{1} \beta_{1} \beta_{2}^{2} \mu_{2}+\beta_{1}^{2} \beta_{2}^{3}+\beta_{1} \beta_{2}^{3} \mu_{2}\right)+\beta_{1} \beta_{2} \mu_{1}\left(\alpha_{1}^{3} \mu_{2}+\alpha_{1}^{2} \beta_{1} \beta_{2}+3 \alpha_{1}^{2} \beta_{2} \mu_{2}+2 \alpha_{1} \beta_{1} \beta_{2}^{2}+3 \alpha_{1} \beta_{2}^{2} \mu_{2}+\beta_{1} \beta_{2}^{3}+\beta_{2}^{3} \mu_{2}\right)+\beta_{1} \beta_{2} \mu_{2}$

$\left(\alpha_{1}^{3} \mu_{1}+3 \alpha_{1}^{2} \beta_{2} \mu_{1}+3 \alpha_{1} \beta_{2}^{2} \mu_{1}+\beta_{2}^{3} \mu_{1}+\alpha_{1}^{2} \beta_{1} \beta_{2}+2 \alpha_{1} \beta_{1} \beta_{2}^{2}+\beta_{1} \beta_{2}^{3}\right)+\alpha_{1} \beta_{1} \beta_{2}\left(\alpha_{1}^{2} \mu_{1} \mu_{2}+\alpha_{1} \beta_{1} \beta_{2} \mu_{1}+2 \alpha_{1} \beta_{2} \mu_{1} \mu_{2}+\right.$

$\left.\beta_{1} \beta_{2}^{2} \mu_{1}+\beta_{2}^{2} \mu_{1} \mu_{2}+\alpha_{1} \beta_{1} \beta_{2} \mu_{2}+\beta_{1}^{2} \beta_{2}^{2}+\beta_{1} \beta_{2}^{2} \mu_{2}\right)+\alpha_{1} \beta_{1} \beta_{2} \mu_{1}\left(\alpha_{1}^{2} \mu_{2}+\alpha_{1} \beta_{1} \beta_{2}+2 \alpha_{1} \beta_{2} \mu_{2}+\beta_{1} \beta_{2}^{2}+\beta_{2}^{2} \mu_{2}\right)+\alpha_{1} \beta_{1} \beta_{2} \mu_{2}$

$\left(\alpha_{1}^{2} \mu_{1}+\alpha_{1} \beta_{1} \beta_{2}+2 \alpha_{1} \beta_{2} \mu_{1}+\beta_{1} \beta_{2}^{2}+\beta_{2}^{2} \mu_{1}\right)$

$D_{2}=\alpha_{1}^{2} \delta_{1} \mu_{2}+\alpha_{1}^{2} \mu_{1} \mu_{2}+\alpha_{1}^{2} \delta_{2} \mu_{1}+\alpha_{1} \beta_{1} \beta_{2} \delta_{1}+2 \alpha_{1} \beta_{2} \delta_{1} \mu_{2}+\alpha_{1} \beta_{1} \beta_{2} \mu_{2}+\alpha_{1} \beta_{1} \beta_{2} \delta_{2}+\alpha_{1} \beta_{1} \beta_{2} \mu_{1}+2 \alpha_{1} \beta_{2} \delta_{2} \mu_{1}+$

$2 \alpha_{1} \beta_{2} \mu_{1} \mu_{2}+\beta_{1} \beta_{2}^{2} \delta_{1}+\beta_{2}^{2} \delta_{1} \mu_{2}+\beta_{1}^{2} \beta_{2}^{2}+\beta_{1} \beta_{2}^{2} \mu_{1}+\beta_{1} \beta_{2}^{2} \delta_{2}+\beta_{1} \beta_{2}^{2} \mu_{2}+\beta_{2}^{2} \mu_{1} \mu_{2}+\beta_{2}^{2} \delta_{2} \mu_{1}$

\section{Results and discussions}

In this section, we numerically obtained the results for mean time to system failure and profit function for all the developed models. For the model analysis, the following set of parameters values are fixed throughout the simulations for consistency:

$\beta_{1}=0.1, \beta_{2}=0.2, \alpha_{1}=0.4, \alpha_{2}=0.1, \delta_{1}=0.1, \delta_{2}=0.3, \mu_{1}=0.3, \mu_{2}=0.4, C_{0}=5,000, C_{1}=1,000$

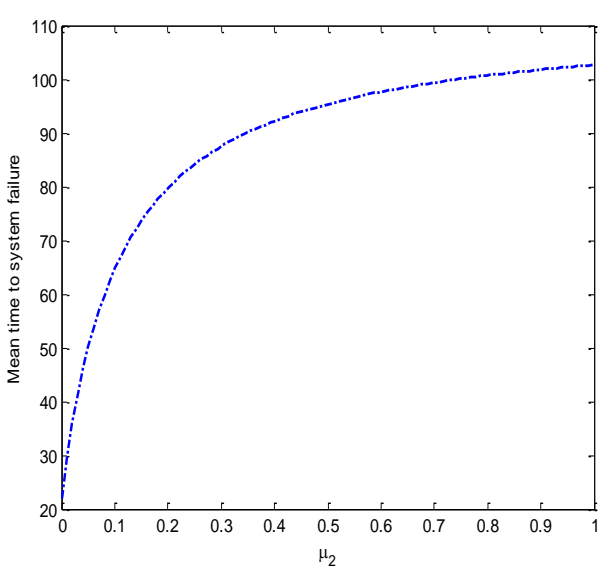

Fig. 2: Effect of $\mu_{2}$ on MTSF

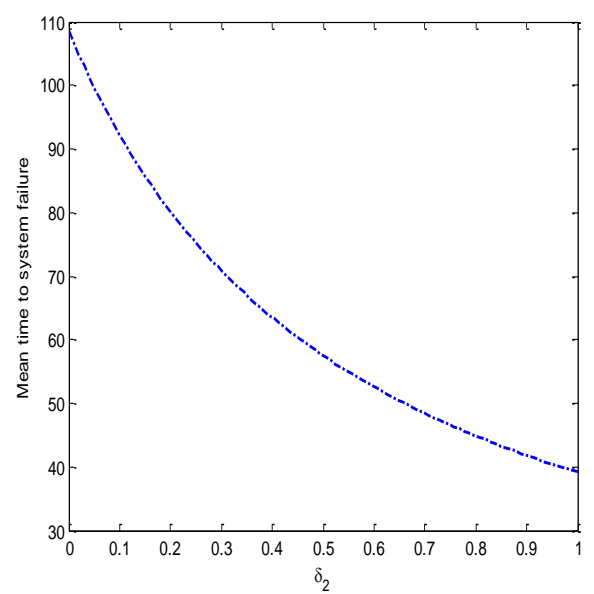

Fig. 4: Effect of $\delta_{2}$ on MTSF

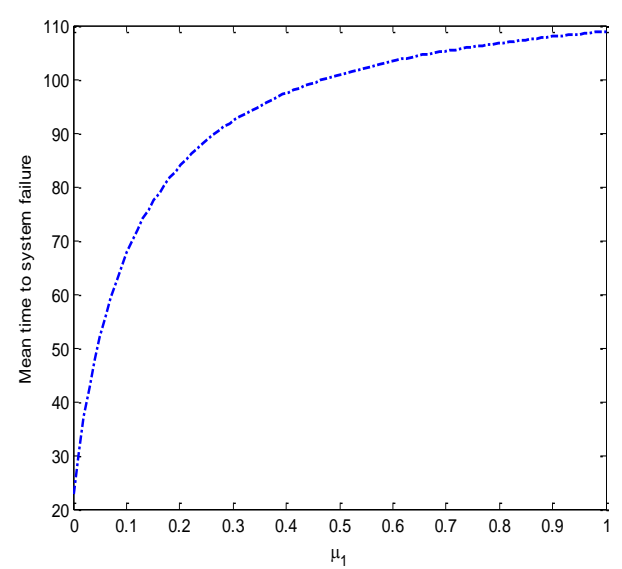

Fig. 3: Effect of $\mu_{1}$ on MTSF

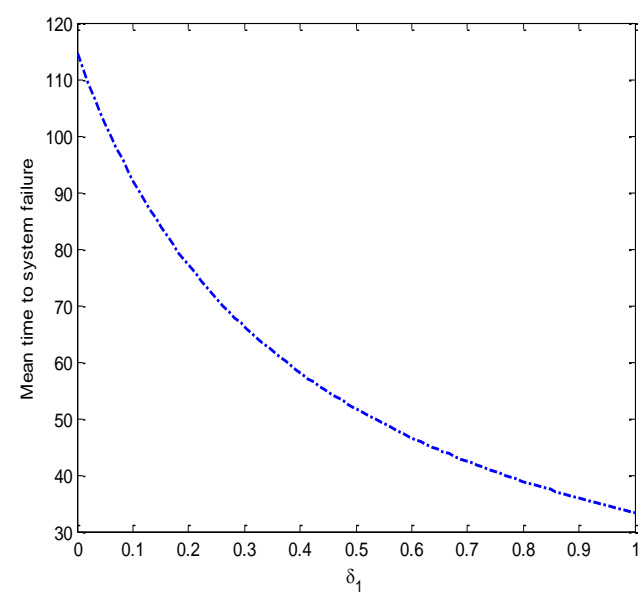

Fig. 5: Effect of $\delta_{1}$ on MTSF 


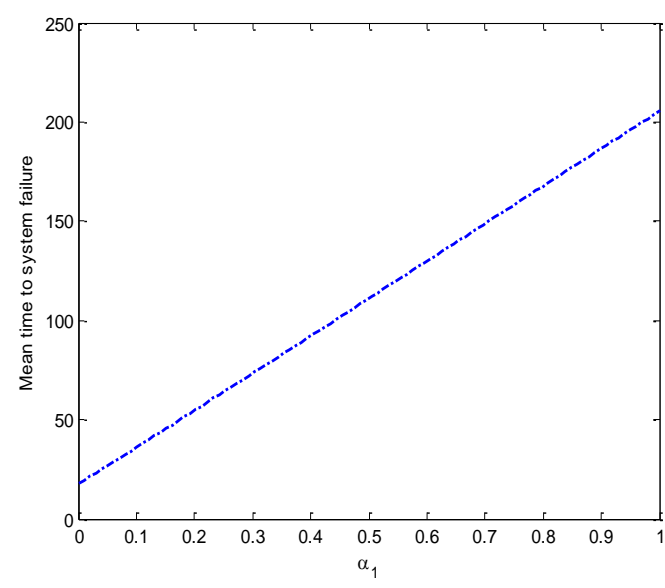

Fig. 6: Effect of $\alpha_{1}$ on MTSF

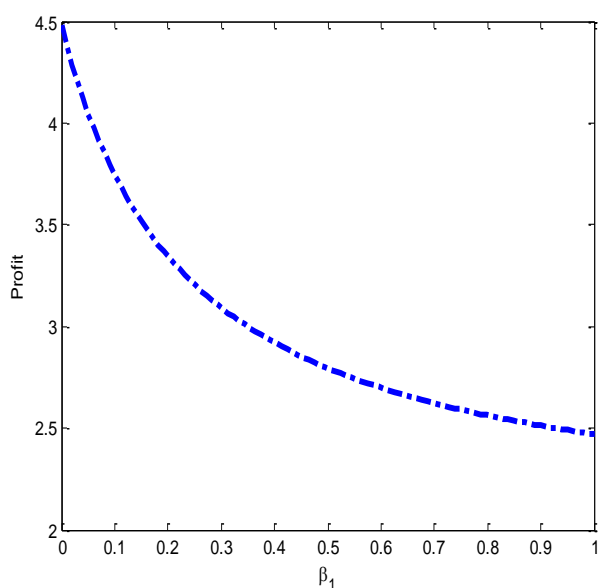

Fig. 8: Effect of $\beta_{1}$ on profit

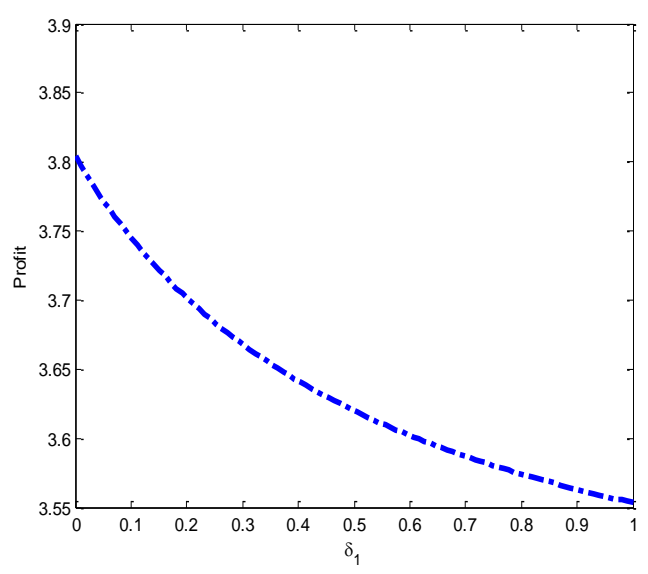

Fig. 10: Effect of $\delta_{1}$ on profit

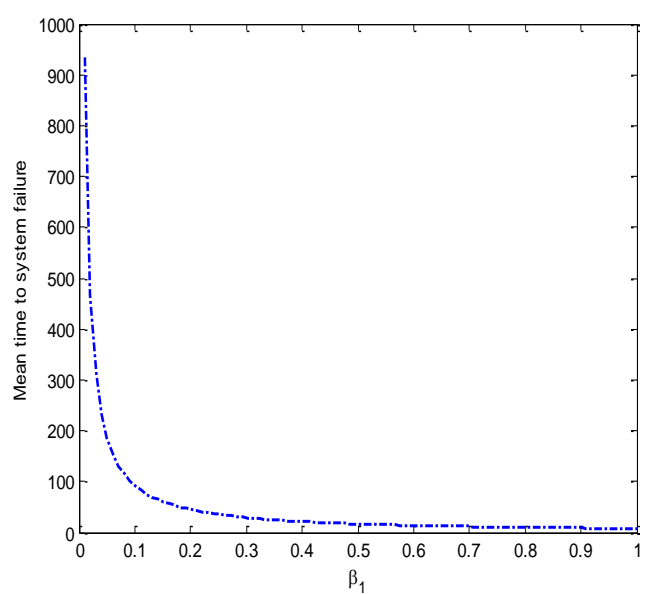

Fig. 7: Effect of $\beta_{1}$ on MTSF

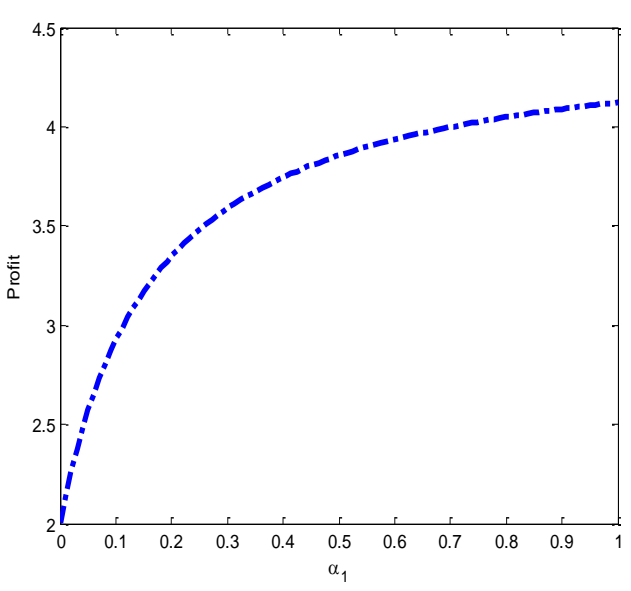

Fig. 9: Effect of $\alpha_{1}$ on profit

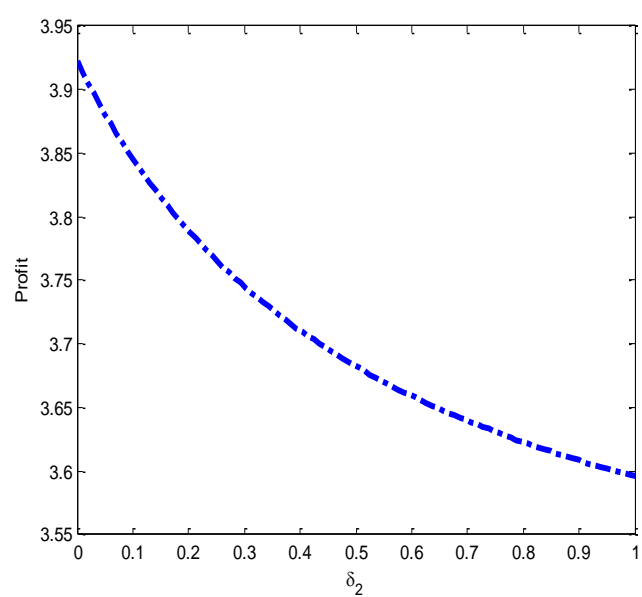

Fig. 11: Effect of $\delta_{2}$ on profit 


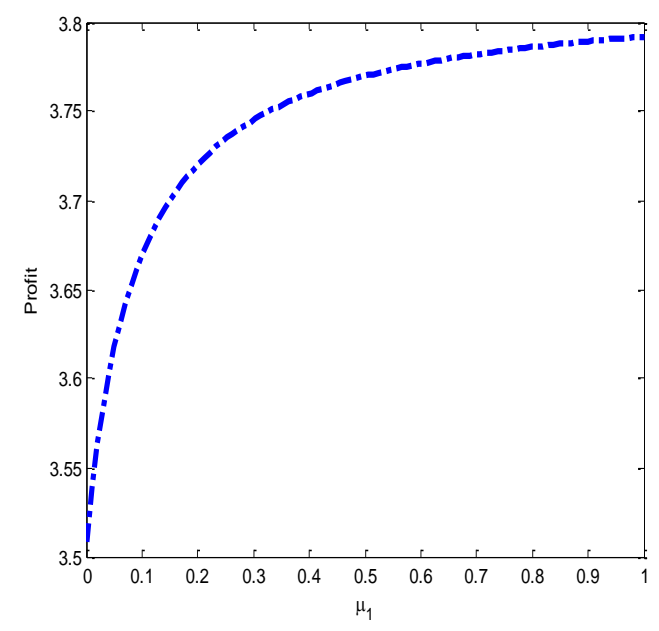

Fig. 12: Effect of $\mu_{1}$ on profit

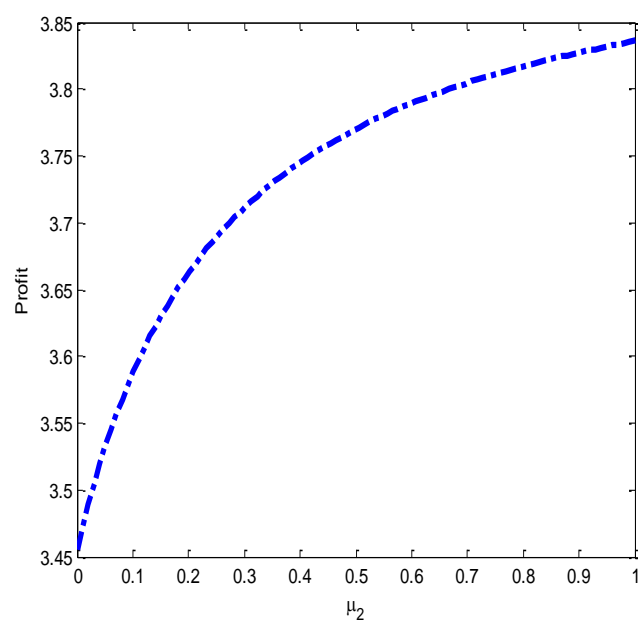

Fig. 13: Effect of $\mu_{2}$ on profit

The simulations in Figures 5 and 10 have shown the impact of slow deterioration $\delta_{1}$ on MTSF and profit. From these Figures it is evident that the MTSF and profit decreases as $\delta_{1}$ increases while in Figures 3 and 12 are corresponding influence of minor minimal maintenance $\mu_{1}$ due to occurrence of slow deterioration on MTSF and profit. In these Figures, it is evident that the MTSF and profit increases with increase in $\mu_{1}$. Similar results can be observed in Figures 4 and 11 for MTSF and profit with respect to fast deterioration $\delta_{2}$. From these figures, the MTSF and profit decreases as $\delta_{2}$ increases and increases with increase in major minimal maintenance $\mu_{2}$ in Figures 2 and 13 respectively. Results of MTSF and profit with respect to $\beta_{1}$ are given in Figures 7 and 8. It is evident from these Figures that as $\beta_{1}$ increases, the MTSF and profit decreases while from Figures 6 and 9, the MTSF and profit increases with increase in $\alpha_{1}$.

\section{Conclusion}

In this paper, we developed the explicit expressions for steady-state availability, busy period, profit function and mean time to system failure of linear consecutive 3-out-of-4 system. In order to determine the effectiveness of the system under study, we performed numerical investigation to see the effect of slow deterioration, fast deterioration, minor minimal maintenance, major minimal maintenance, failure and repair rates on mean time to system failure and profit. It is evident from the results obtained that repair rate, minor minimal maintenance rate and major minimal maintenance rates increase the meant time to system failure and profit generated while slow deterioration, fast deterioration and failure rate decreases the mean time to system failure and profit.

\section{References}

[1] R.K. Bhardwaj and S. Chander. Reliability and cost benefit analysis of 2-out-of-3 redundant system with general distribution of repair and waiting time. DIAS- Technology review- An Int. J. of business and IT. 4(1), 2007, pp 28-35.

[2] S. Chander and R.K.Bhardwai Reliability and economic analysis of 2-out-of-3 redundant system with priority to repair. African J. of Maths and comp. sci, 2(11), 2009, pp 230-236.

[3] R.K. Bhardwai and S.C. Malik. MTSF and Cost effectiveness of 2-out-of-3 cold standby system with probability of repair and inspection. Int. J. of Eng. Sci. and Tech. 2(1), 2010, pp 5882-5889.

[4] K. Wang, C.Hsieh and C. Liou, Cost benefit analysis of series systems with cold standby components and a repairable service station. Journal of quality technology and quantitative management, 3(1), 2006, pp 77-92.

[5] K.M. El-Said. Cost analysis of a system with preventive maintenance by using Kolmogorov's forward equations method. American Journal of Applied Sciences 5(4), 2008, pp 405-410.

[6] M.Y.Haggag. Cost analysis of a system involving common cause failures and preventive maintenance. Journal of Mathematics and Statistics 5(4), 2009, pp 305-310.

[7] K.H. Wang and C.C. Kuo. Cost and probabilistic analysis of series systems with mixed standby components.Applied Mathematical Modelling, 24, 2000, pp 957-967.

[8] K.C. Wang, Y.C, Liou and W.L. Pearn. Cost benefits analysis of series systems with warm standby components and general repair time. , Mathematical Methods of operation Research, 61, 2005, pp 329-343.

[9] I. Yusuf. Availability and profit analysis of 3-out-of-4 repairable system with preventive maintenance, International Journal of Applied Mathematical Research, Vol. 1, No.4, 2012,510-519.

[10] H.S. Fathabadi and M. Khodaei. Reliability evaluation of network flows with stochastic capacity and cost constraint, Int. J. of Mathematics in Operational Research, Vol.4, No.4, 2012, pp $439-452$. 